\title{
Aerodynamic adaptivity criterion in the production methodology of the energy-efficient mine turbomachines
}

\author{
Vladimir Makarov *, Nikolai Makarov, Alexandr Lifanov, Artem Materov, and Nikolay \\ Kosarev
}

Ural State Mining University, 620144 Ekaterinburg, Russia

\begin{abstract}
Competitive growth of enterprises in the mining and oil and gas industries of the Russian economy, combined with industrial safety requirements, updates the task of developing the design and production methodology for the aerodynamically adaptive turbomachines with a nature-like dominance. Such machines adequately and economically soundly establish the necessary parameters of the air environment in the technological space that implement the concept of optimal subsurface management ecotechnology. This article proposes a production methodology for the energy-efficient turbomachines using the aerodynamic adaptability criterion that determines the relations between the velocity circulation and flow acceleration around the vane cascade profiles as the nature-like dominance of the process for converting the mechanical rotational energy of impeller into the internal energy of the air flow. The Karman theory of bound and free vortices, the ChaplyginJoukowski-Kutta hypothesis, the conformal mapping method, and curve irregularities are used for development of a mathematical model for controlling the aerodynamic adaptability. It is proved that the control dominant is the intensity of the sources distributed over the turbomachine impeller vane profile that determine the flow diffusivity and as a result the acceleration circulation around the profile. It has been experimentally confirmed that the use of profiles obtained using the proposed technique increases the aerodynamic adaptability coefficient of the turbomachine by $51 \%$, while increasing the area of its cost-effective performance by at least 2 times.
\end{abstract}

\section{Introduction}

The environmental and industrial safety protection of enterprises of the mining and oil and gas complex of the Russian Federation is equivalent to $13 \%$ of the country's GDP. Significant changes in the aerodynamic parameters of the process environment impair the energy efficiency of turbomachines due to its low adaptability [1-4]. Competitive growth of enterprises combined with the introduction of environmental technologies updates the task of developing the design and production methodology for the aerodynamically

\footnotetext{
* Corresponding author: mnikolay84@mail.ru
} 
adaptive turbomachines that adequately and economically soundly establish the necessary air parameters in the technological space that implement the concept of optimal innovative subsurface management.

The implementation of nature-likeness standards in relation to the turbomachines is based on establishment of the dominant mechanism of interaction between the turbomachine impeller blade row and air flow, the control of which allows to respond to the external factors determined by the technological production process adequately and with minimal energy losses.

This article proposes a production methodology for the energy-efficient turbomachines using the aerodynamic adaptability criterion that determines the relations between the velocity circulation and flow acceleration around the vane cascade profiles as the naturelike dominance of the process for converting the mechanical rotational energy of impeller into the internal energy of the air flow.

\section{Statement of the problem}

The interaction of the air flow with the turbomachine impeller blades is performed through a stable Karman vortex street formed by the bound vortex system being a hydrodynamic analogue of the blade profile [5-7].

When the aerodynamic parameters of the process environment are changed, the vortex system is deformed, broken, and as a result, the intensity of the bound vortices decreases being a hydrodynamic analog of the velocity circulation, that is, the pressure distribution according to the Ostrogradsky - Gauss theorem [7-9]. Simultaneously with a decrease in circulation, there is an increase in pressure losses for the separated vortex generation due to the occurrence of local diffusivity, velocity gradients, that is, a sharp increase in the negative flow acceleration circulation.

According to the results of the studies, Figure 1c shows the pressure distribution over the blade profile (lines $a, b, c)$ depending on the position of the flow separation point $(A, B$, $C$ ), i.e., its rear stagnation point (RSP) (Fig. 1a). As the angle of attack increases from the negative to positive values, the RSP $(C)$ moves from the working surface to the back surface $(B)$, while changing the pressure distribution on the profile, significantly affecting the velocity circulation and flow acceleration around it and, as a result, the aerodynamic quality of the blade cascade. The RSP position affects the downstream flow angle. The significant changes in the downstream flow angle with a relatively small changes in the RSP position indicate that even with the potential classical profile flow in the cascade, the velocity and acceleration circulation created is determined not only by the selection of the profile configuration downstream flow angle, but to a large extent by the RSP position that does not match the profile back edge.

On line $a$ (Fig. 1b), the RSP coincides with the exit edge of the blade profile. The flow is moving along the profile towards the RSP with the smooth and monotonous changes, without increasing the acceleration circulation to the values in the RSP. It eliminates the separated vortex generation and, accordingly, ensures high values of aerodynamic quality.

On line $b$ (Fig. 1b), the RSP is shifted toward the rarefaction region. For this reason, the flow moving along the profile is rapidly slowing down, and its zero speed is achieved earlier than the profile back edge is actually located. Moreover, in the area of high pressure, the flow passing through the high curvature section significantly increases the velocity due to the negative pressure gradient. After flowing around the high curvature region, the flow sharply slows down, and the large positive pressure gradients occur prior to the actual RSP position. In this case, the pressure and velocity distribution diagrams on the profile working and back surfaces intersect at a point located upstream of the RSP. It causes the separated vortex generation and a decrease in its aerodynamic quality. 
On line $c$ (Fig. 1b), the flow vanishing point is located in the area of increased pressure. Moreover, it is in this region that the sharp gradients of positive pressure occur, with a decrease in the flow velocity that leads to an early flow downstream from the profile. In the rarefaction region relevant to the high profile curvature, the large velocity values are reached, after which there is a sharp flow deceleration, as on the line $b$ of the high-pressure region.

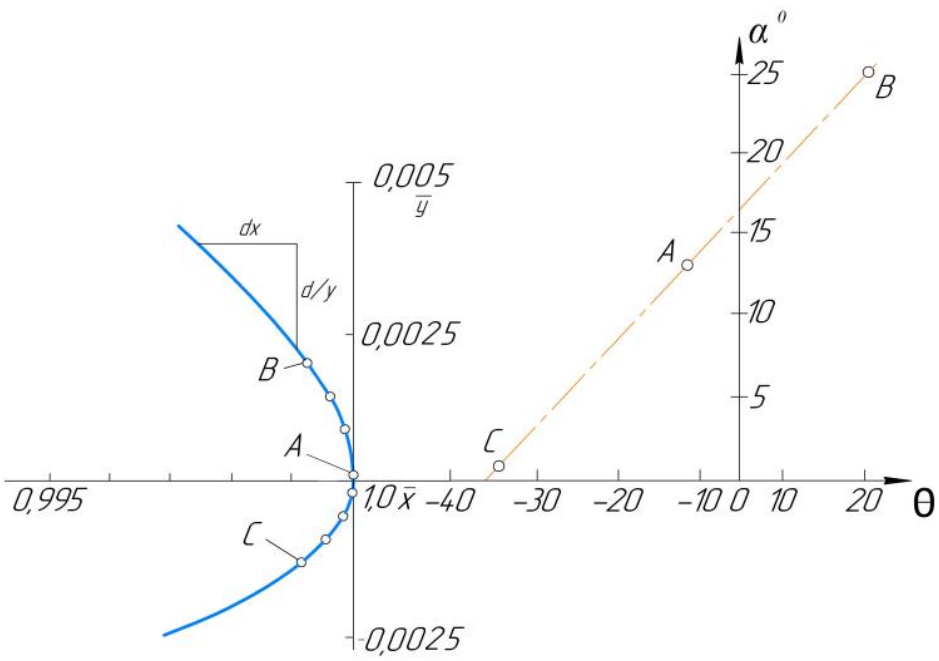

$a$

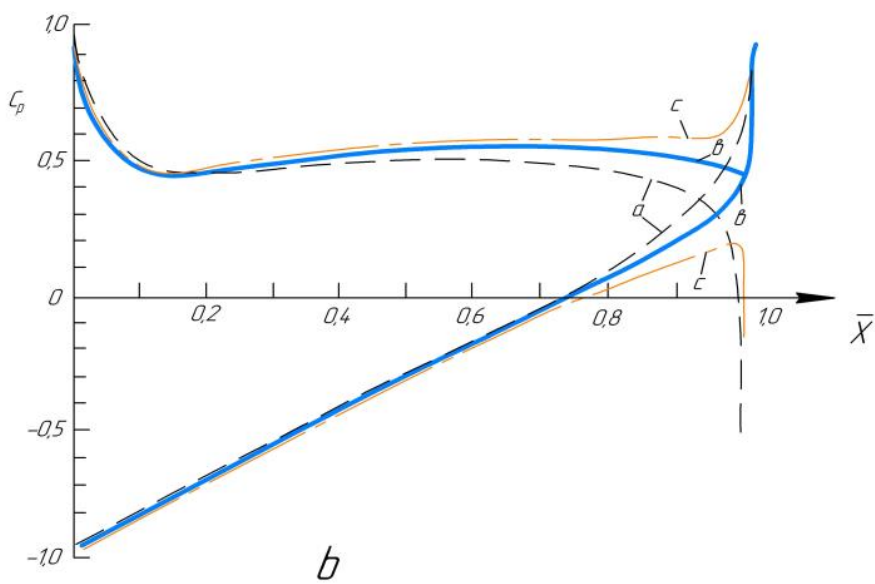

Fig. 1. Influence of the rear stagnation point position on the flow diffusivity around the profile: a) Position of the rear stagnation point at the profile output and changes in the downstream flow angle; b) Pressure coefficient distribution that is a hydrodynamic analog of local diffusivity on the profile surface.

The similar results of the pressure distribution dependence on the blade profiles and the RSP position were obtained in the papers [10-12].

Thus, the RSP position, ceteris paribus, significantly affects the pressure and velocity distribution in the turbomachine impeller blade cascades, that is, its aerodynamic quality. 
The local velocity change based on the pressure gradient is more than the permissible value, that is, a negative acceleration circulation leads to the flow deceleration to a speed equal to 0 . It is the RSP shift in relation to the profile back edge.

Thus, the acceleration circulation on the blade profile, even in the conditions of potential flow, causes the flow separation phenomenon that is dominated by the local diffusivity determined by the following formula:

$$
K_{q}^{\mathrm{\pi}}=\frac{\partial \bar{y}}{\partial \bar{x}}=-\frac{\partial \bar{V}}{\partial \bar{x}}
$$

where $\bar{y}, \bar{x}$ are the relative coordinates of the blade profile points in the turbomachine impeller cascade; $\bar{x}=x / b ; \bar{y}=y / b ; b$ - blade chord, $\mathrm{m} ; \bar{V}$ - relative air flow rate on the blade profile; $\bar{V}=V / u ; u$ - blade speed, $\mathrm{m} / \mathrm{s}$.

It follows from (1) that for the classical blade profiles of the turbomachine impellers there is an internal contradiction between the need to increase the positive velocity gradient for the circulation growth that determines the pressure, i.e. the level of energy interaction to the maximum velocity point, and provision of the maximum permissible negative velocity gradient, reflecting the hydrodynamic analogy with the local diffusivity. In this case, under the potential flow conditions, the separated vortex generation and displacement of the RSP profile occur in relation to the profile back edge (see Fig. 1).

Therefore, the above facts are fundamentally important for obtaining the maximum aerodynamic load of turbomachines in its nominal operating mode that ensures the highest efficiency. However, given the high requirements for the mine turbomachine adaptability, this is even more important due to the wide changes in their operating modes related to the changes in the process parameters.

Having the high efficiency and aerodynamic loading in the nominal mode, as shown above, the turbomachines sharply reduce economic efficiency when changing the external conditions due to the changes in the kinematic parameters of flow around the impeller blade profile.

\section{Research design}

The design features of turbomachines make it possible to implement the active vortex circulation control methods using the methodology of external sources and vortices that form the variable-shape aero-gas-dynamic profiles by energetically affecting the streamline shapes and profile flows.

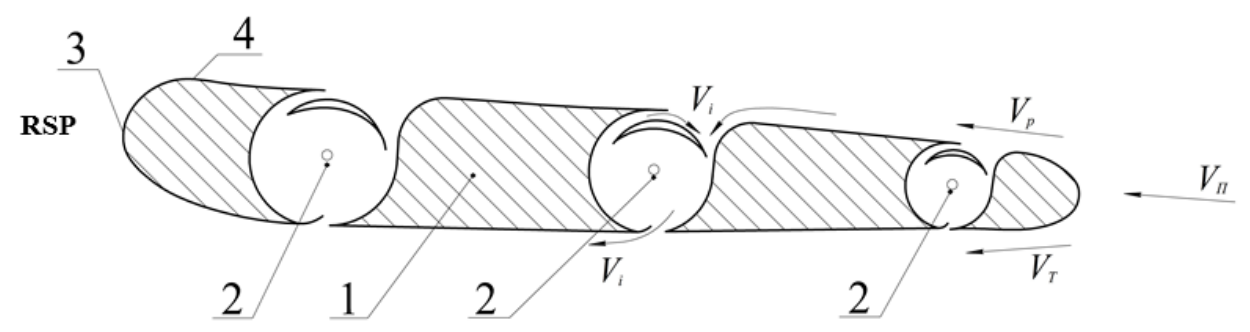

Fig. 2. Turbomachine impeller blade profile with a device for controlling the Karman vortex street.

The jets of sources 2, the energy parameters $V_{i}$ of which are interrelated with the external network specifications, slow down or accelerate the air velocity $V_{\mathrm{p}}, V_{\mathrm{T}}$ on the 
working and back surfaces of the blade profile 1, combining the RSP 3 and the flow separation point 4 , changing the velocity and flow acceleration circulation and as a result, the aerodynamic specifications of the turbomachine adaptively to the external conditions. The energy parameters of the bound vortices, formed by the jets of sources 2 , the position of the flow separation point and the RSP in the aero-gas-dynamic profiles are determined by the source specification and its feedback with the changing external conditions.

The stable feedback of vortex chambers, Karman vortex street control devices, the technical possibility of its use in the mathematical model generation that determines the increased adaptability of turbomachines, have not yet been considered during the turbomachine design and production. In this case, the force interaction of the impeller blades and air flow occurs through an adaptive aerodynamic vortex system that ensures the efficiency of turbomachines and its susceptibility to the changes in the external environment. Just as the bird's wing provides an efficient continuous flowing round while changing its shape during interaction with the air flow, the bound vortex system created by the external sources of the aero-gas-dynamic profile of the turbomachine impeller blades, being a mediator in the interaction of the blade channel air flow with the blades, also provides its continuous flowing round. The turbomachines designed on the basis of this principle are distinguished by the fact that a wide range of changes in the external conditions is nonetheless a nominal effective operating mode.

Control over the profile cascade rotational flow by the external sources increases its aerodynamic loading, reduces energy losses and, as a result, increases adaptability. It shall be emphasized that these two processes are interconnected. The main task in the development of active flow control methods in the blade cascades is to establish the energy parameters of external sources, at which the separated vortex generation is eliminated and, as a result, the circulation is achieved. It means the level of energy interaction relevant to the potential ideal gas flow, the RSP combination with the profile back edge.

In order to develop the production methodology for turbomachines that provide the highest possible energy efficiency for a given field of aerodynamic parameters, a scientific hypothesis is proposed. The dominant feature of controlling the aerodynamic adaptability of turbomachines functionally related to the aerodynamic quality of the impeller blade cascades, as the main element for the aerodynamic parameter formation, is the ratio of the acceleration and velocity circulation around the impeller blade profiles.

Having considered the principle of hydrodynamic analogy of the conformal mapping method (1), Fig. 1, we obtain the formula for calculating the local aerodynamic adaptability coefficient in the conditions of air flow along the profile line:

$$
K_{\mathrm{a}}^{\text {Л }}=1-\frac{d \gamma}{d t} \gamma^{-1} \omega^{-1},
$$

where $\gamma$ is the air flow circulation around the blade profile in the turbomachine impeller cascade; $\omega$ - angular velocity of the relative profile motion, $\mathrm{c}^{-1}$.

While using the graphical model shown in Fig. 2, we obtain the flow potential on a multivalent Riemannian domain which parameters are the functions of the external network parameters $[2,3]$.

Let us develop a mathematical model for calculating the aerodynamic adaptability coefficient $[4,8,9,10]$. 


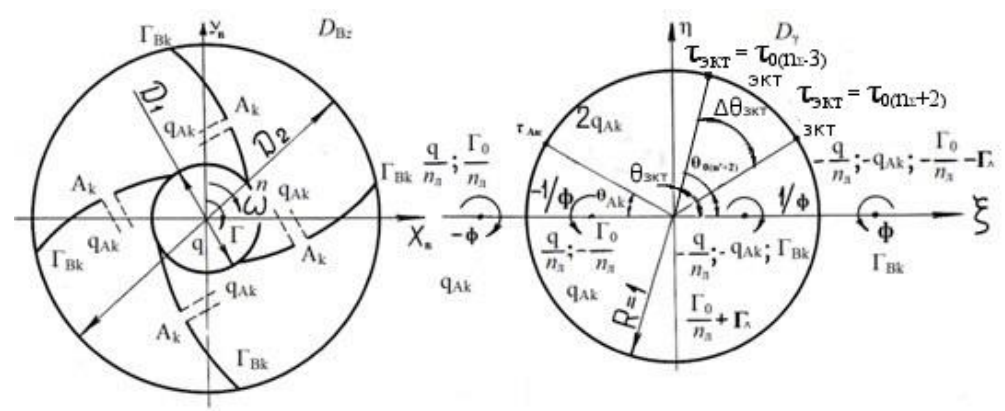

Fig. 3. Conformal mapping of the blade cascades with the Karman vortex street control devices and critical point indication.

The aerodynamic adaptability coefficient (2) on the multivalent Riemannian domain can be represented as follows:

$$
F_{\theta}\left(K_{\mathrm{a}}^{\text {л }}\right)=K_{\theta}^{\text {д }}=1-\frac{d \rho}{d t} \rho^{-1} \omega^{-1},
$$

where $F\left(K_{\mathrm{a}}^{\mathrm{\pi}}\right)$ is the function of the flow conformal mapping around the blade profile in the turbomachine impeller cascade to the exterior of the unit circles of the multivalent Riemannian domain $[5,10] ; \rho$ - air flow circulation around the profile on the multivalent Riemannian domain; $F_{\theta}(\theta)=y(x) ; F_{\theta}(\rho)=\gamma[5,8,10]$.

Having considered the Kelvin's theorem with the closed profile circuit, we obtain a calculation formula for the aerodynamic adaptability coefficient in the Riemannian domain $[8,10]$ :

$$
K_{\theta 1}^{\theta_{\mathrm{RSP}}}=1-\int_{\theta 1}^{\theta_{\mathrm{RSP}}} \frac{d \rho}{d \theta} \rho^{-1} d \theta,
$$

$\theta$ the vectorial angle of the blade point position in the classical multivalent Riemannian domain; $\theta_{1}, \theta_{\mathrm{RSP}}$ - the vectorial angle of the forward and rear stagnation points.

The complex potential from the sources with intensity $q$ will be obtained in the following form $[6,8,10]$ :

$$
\varphi_{q_{i}}=\frac{q_{i}}{\pi}\left[\ln \left(\theta-\theta_{i}\right)-\ln \left(\theta^{2}-P\right)-\ln \left(\theta^{2}-P^{-2}\right)\right],
$$

where $P$ is the form parameter of the equivalent blade cascade in the form of logarithmic spirals; $q_{i}$ - intensity of sources located at the profile points determined by the angles $\theta_{i}$.

The complex potential from the vortex in the RSP is obtained in the following form [8, 10]: 


$$
\varphi_{\rho_{\mathrm{B}}}=\frac{\rho_{\mathrm{B}}}{2 \pi i} \ln \frac{\theta_{\mathrm{RSP}}-\rho^{-1}}{\theta_{\mathrm{RSP}}-P},
$$

where $\rho_{\mathrm{B}}$ is the circulation (vortex intensity) in the RSP on the multivalent Riemannian domain that determines the RSP shift for the angle of $\Delta \theta_{\text {RSP }}$.

Having considered [6] and (4-8), the flow velocity in the Riemannian domain is obtained as follows:

$$
\begin{aligned}
& F_{\theta}(V)=\frac{d \varphi}{d \theta}=\frac{q\left(\theta+P-\frac{1}{\theta-P}\right)}{\pi n_{\text {л }}}+\frac{\left(q-n_{\text {л }} \sum_{i=1}^{n} g_{i}\right)}{2 \pi n_{\text {л }}\left(\theta_{i}+P\right)}+\frac{\left(q-n_{\text {л }} \sum_{i=1}^{n} q_{i}\right)}{2 \pi n_{\text {л }}\left(\theta_{i}+P^{-1}\right)}+ \\
& +\frac{i n_{\text {л }} \rho+i n_{\rho} \rho_{\mathrm{RSP}}-n_{\text {л }} \sum_{i=1}^{n} g_{i}-g}{2 \pi n_{\text {л }}\left(\theta_{i}+P\right)}-\frac{i n_{\text {л }} \rho+i n_{л} \rho_{\mathrm{RSP}}+n_{\text {л }} \sum_{i=1}^{n} q_{i}+q}{2 \pi n_{\text {л }}\left(\theta_{i}+P^{-1}\right)}+\frac{\sum_{i=1}^{n} q_{i}}{\pi\left(\theta-\theta_{i}\right)},
\end{aligned}
$$

where $n_{\text {л }}$ is the number of profiles in the cascade.

Having considered (4), the equation for the aerodynamic adaptability coefficient is obtained in the following form:

$$
\begin{aligned}
K_{a}=1-\int_{\theta_{1}}^{\theta_{\mathrm{RSP}}} \frac{4 P g_{i}\left(\theta_{i}\right)\left(P^{4}-1\right) \cdot}{q_{i}\left[\frac{\operatorname{Sin}\left(\theta_{i}-\theta_{3}-\Delta \theta_{\mathrm{RSP}}\right)}{1-\operatorname{Cos}\left(\theta_{i}-\theta_{3}-\Delta \theta_{\mathrm{RSP}}\right)}-4 P\left(P^{4}-1\right) \frac{\operatorname{Sin}\left(\theta_{3}+\Delta \theta_{\mathrm{RSP}}\right)}{P^{2}+2 P \operatorname{Cos}\left(\theta_{3}+\Delta \theta_{\mathrm{RSP}}\right)+1}\right]+} \\
+\frac{\cdot\left[\operatorname{Cos} \theta_{i}\left(P^{2}+1-2 P \operatorname{Cos} \theta_{i}\right)-\operatorname{Sin} \theta_{i}\left(P^{2}+1+2 P \operatorname{Sin} \theta_{i}\right)\right]}{{ }^{2 P \operatorname{Sin}\left(\theta_{3}+\Delta \theta_{\mathrm{RSP}}\right)}}-4 P q \frac{\left(P^{2}+1\right) \operatorname{Sin}\left(\theta_{3}+\Delta \theta_{\mathrm{RSP}}\right)}{P^{2}-1}+\rho_{\mathrm{B}} \frac{\operatorname{Sin} \Delta \theta_{\mathrm{RSP}}}{1-\operatorname{Cos} \Delta \theta_{\mathrm{RSP}}}
\end{aligned}
$$

\section{Results and discussion}

The use of the conformal mapping and the Chaplygin singularity method, having considered the proposed hypothesis about the relations between the acceleration circulation around the turbomachine impeller cascade profiles and the energy losses when changing the external factors, has made it possible to obtain a mathematical model for controlling the aerodynamic adaptivity coefficient. The dominant control feature is the intensity of distribution of sources to the profiles $q_{i}$, that is determined by the condition of ensuring minimum diffusivity values. The vortex intensity $\rho_{\mathrm{B}}$ is determined by the condition of the RSP combination with the flow separation point. The proposed mathematical model (8) leads to the conclusion that it is necessary to produce the blade profile with a rounded back edge (Fig. 1,2) for the RSP position smooth control. As shown above, it has a significant effect on the velocity and flow acceleration circulation. The profiles with rounded back edges are currently used on the highly maneuverable aircrafts [10].

The proposed hypothesis allows to solve the problem of designing the turbomachine impeller blade cascade with the maximum adaptability in a given range of operating modes $\Delta q=q_{\max }-q_{\min }$, as the main purpose indicator for the classical minimax problem in the 
potential flow conditions, that is, determination of the optimal value of the parameters $P(q), q(\theta), \rho_{\mathrm{B}}(\theta)=f(q)$ based on the condition for the maximum functional value (8). In principle, there are three possible practical solutions for designing the turbomachines with aero-gas-dynamic profiles:

1. Determination of the minimum value of energy losses due to the optimal distribution of the distributed sources intensity $q(\theta)$ in the region of diffuse flow on the blade profile to ensure the minimum allowable diffusivity $K_{q}^{a}$ in conditions of the fixed circulation $\rho$ and pressure $\psi$ by controlling the RSP position $\left(\theta_{\text {RSP }}\right)$ using a vortex with the intensity $\rho_{\text {в }}$ :

$$
K_{q}^{a}=\frac{\frac{d \varphi}{d q}}{\frac{d \varphi}{d \theta}+\frac{d^{2} \varphi}{d \theta^{2}} d \theta+q(\theta) d \theta}
$$

2. Determination of the maximum circulation flow around the blade cascade, that is, the largest pressure value at a fixed acceleration circulation value relevant to the energy losses for the vortex generation.

3. Determination of the maximum aerodynamic adaptability coefficient value $K_{A}$ by minimizing the acceleration circulation with the maximum velocity circulation value on the blade profile, determined as follows [2-4, 9]:

$$
K_{A}=\frac{\int_{q_{\min }}^{q_{\max }} K_{\mathrm{a}} d q}{\int_{q_{\min }}^{q_{\max }} d q} \rightarrow \max
$$

In order to confirm the reliability of the proposed hypothesis, having considered [24.8], an aerodynamic bench meeting the requirements of GOST 10921-90 was used for testing the classical and aerodynamic blade cascade. It was considered that the velocity and acceleration circulation around the blade cascade, the aerodynamic adaptability criterion, and the air flow rate coefficient are the hydrodynamic analogues of the lift force coefficient $C_{y}$, profile drag coefficient $C_{x}$, aerodynamic quality coefficient $K=\frac{C_{y}}{C_{x}}$ and attack angle coefficient $\alpha[6,8]$.

Figures 4 and 5 show the graphs of variances in the profile drag coefficient and the lift force coefficient of the blade cascade. They confirm that control over the Karman vortex street using the sources allows to significantly reduce energy losses and increase the pressure developed by the turbomachine especially in the off-design modes. 


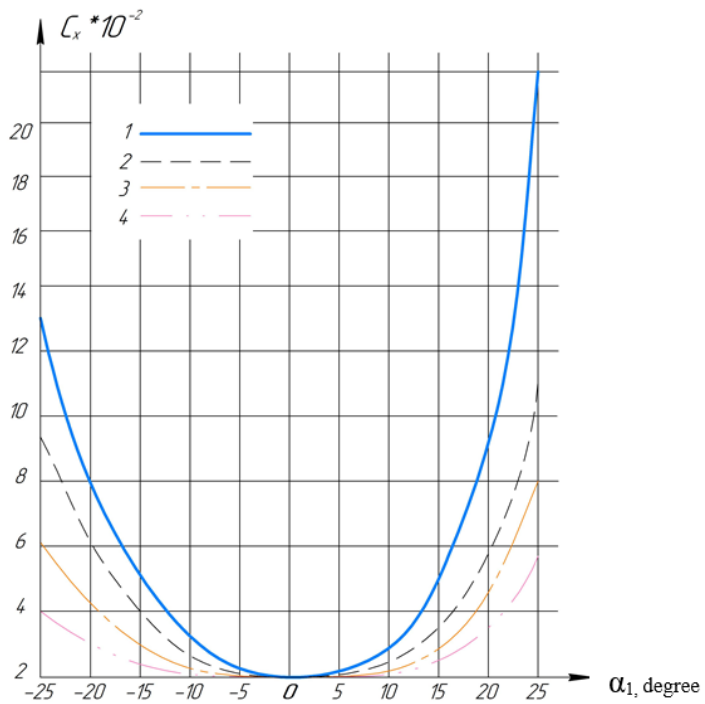

Fig. 4. Dependence of the profile drag coefficient of the lift force that is a hydrodynamic analogue to the acceleration circulation of the turbomachine blade profile from the angle of attack: 1 - classic profile, 2 - profile with a vortex source in the RSP, 3 - profile with a vortex source in the RSP and FSP, 4 - profile with the distributed sources along the blade profile.

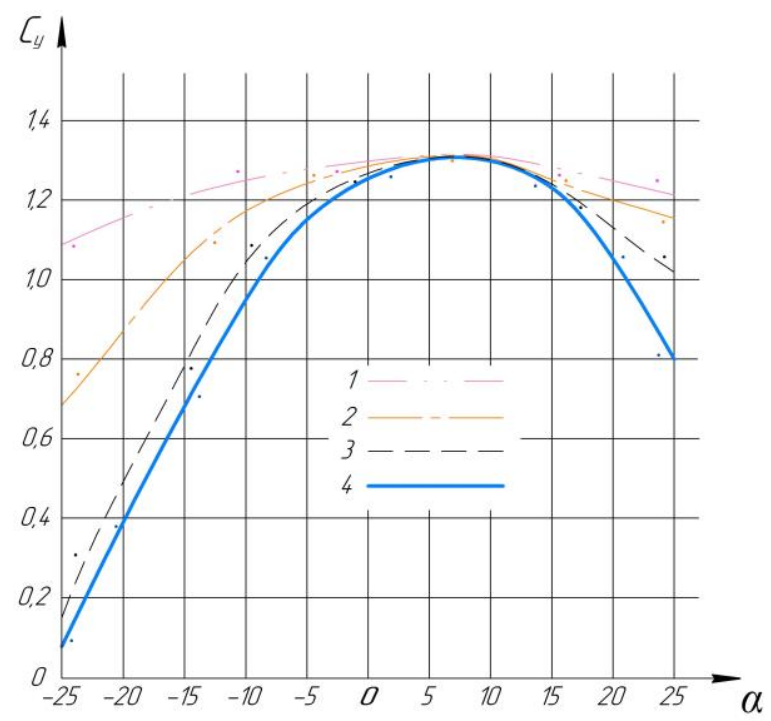

Fig. 5. Dependence of the lift force coefficient that is a hydrodynamic analogue of the velocity circulation of the turbomachine blade profile from the angle of attack: 1 - classical profile, 2 - profile with a vortex source in the RSP, 3 - profile with a vortex source in the RSP and FSP, 4 - profile with the distributed sources along the blade profile.

The analysis of the blade cascade test results given in Fig. 4, 5 shows that the greatest efficiency of controlling the profile drag, lift force and aerodynamic quality of the blade cascade with the minimal complication of the impeller design is achieved by using the distributed sources for the Karman vortex street control along the blade profile on its 
working surface in the region of negative thickness gradients.

In the angle-of-attack range of $-25^{\circ}-25^{\circ}$, corresponding to the changes in the air flow rate coefficient from 0.2 to 2.2 , the profile drag coefficient is decreased by 3.6 times, the lift force coefficient is increased by $34 \%$, the aerodynamic quality coefficient is increased by $47 \%$. At the same time, the aerodynamic adaptability criterion calculated according to the proposed model is increased by $51 \%$ from 0.65 to 0.89 that allows a 2 -fold increase in the area of cost-effective performance of turbomachines.

\section{Conclusions}

1. The hypothesis is proved on a theoretical and experimental level that the dominant control of the turbomachine aerodynamic adaptability is the flow acceleration circulation around the impeller blade cascades in relation to its velocity circulation acting as the hydrodynamic analogues of the profile drag and lift force.

2. The proposed hypothesis is used to develop a mathematical model in the form of a functional, the maximum value of which determines the optimal parameters of aerodynamic profiles with the Karman vortex street control devices that provide the greatest aerodynamic adaptability of the turbomachine.

3. The vortex sources at the leading and exit edges of the blade cascades and a distributed source at the output section of its working surface allow to increase the aerodynamic adaptability coefficient of the turbomachine from 0.65 to 0.89 , that is, to expand the field of its cost-effective performance by 2 times.

\section{References}

1. N. V. Makarov, V. N. Makarov, I. A. Volegzhanin, A. V. Ugolnikov, Mining Information and Analytical Bulletin of the Moscow State Mining University, 5, 171 (2018)

2. S. Ya. Davydov, N. G. Valiev, N. V. Grevtsev, L. N. Oleinikova, Sustainable Development of Mountain Territories, 11(3), 273 (2019)

3. N. V Makarov, V. N. Makarov, E. E. Franyuk, Conf. Ser.: Earth Environ. Sci. 272 032075 (2019)

4. S. Y. Davydov, N. P. Kosyrev, N. G. Valiev, D. I. Simisinov, V. A. Kurochkin, A. E. Zamuraev, Refractories and Industrial Ceramics, 54 (3), 178 (2013)

5. N. V Makarov, V. N. Makarov, E. E. Franyuk, IOP Conf. Ser.: Earth Environ. Sci. 272, 032075 (2019)

6. N. V. Makarov, V. N. Makarov, A. V. Lifanov, A. V. Ugolnikov, V. M. Tauger, Mining Information and Analytical Bulletin, 10, 206 (2019)

7. Wang P. Mulyi-Objective design of a transonic turbocharger compressor with reduced Noise and increased efficiency, (2017)

8. L. G. Loytsyansky, Mechanics of fluid and gas. College textbook, 840 (2013)

9. S.A.M. Torshizi, A. H. Benisi, and Durali, M. Numerical, ASME Turbo Expo 2016: Turbomachinery Technical Conference and Exposition, Seoul, 1 (2016)

10. D. Zh. Gostelow, Aerodynamics of lattices of turbomachines, 391 (1987)

11. S.A.M. Torshizi, A.H. Benisi, and M. Durali, ScientiaIranica, 24, 707 (2017)

12. MAO Y. F., Numerical Study of Correlation between the Surge of Centrifugal Compressor and the Piping System. Ph.D. Thesis, Xi'an Jiaotong University, Xi'an. (2016) 